\title{
Transverse Testicular Ectopia with Persistent Mullerian Duct Syndrome: Presentation and Management
}

\author{
Aashish Rajesh $^{1}$ (D) Mohammed Farooq ${ }^{2} \cdot$ Hemashree Rajesh $^{3}$ \\ Received: 27 May 2019 / Accepted: 14 February 2020 / Published online: 24 February 2020 \\ (C) Association of Surgeons of India 2020
}

Sir,

We appreciate the authors' contributions in the report titled Hernia Uteri Inguinale: a Travesty of Nature [1]. The authors described a case of transverse testicular ectopia (TTE) with persistent Mullerian duct syndrome (PMDS) in a 30-year-old male patient managed with resection the Mullerian structures and orchidopexy.

It is remarkable to note that an individual with just a single left-sided cryptorchid testis had a sperm count of 35 million/ml (normal range) and that the patient's fertility hormones were within normal limits. A 30-year cryptorchid testis is likely to have severe atrophy with minimal testosterone production and the resulting lack of feedback inhibition would cause an increased level of both folliclestimulating and luteinizing hormones. We would be interested to know the authors' rationale for not performing a pre-operative or intra-operative testicular biopsy prior to opting for orchidopexy. Jeong et al. found that almost all patients in their cohort with bilateral testicular pathology were azoospermic [2]. The results of the testicular biopsy could have enabled identification of potential malignancy and may have changed management.

The authors discussed two anatomic variants of PMDS. There are, however, three clinical presentations of PMDS [3]:

1. Testes in anatomical position of ovaries with an empty inguinal sac

Aashish Rajesh

rajesh.aashish@mayo.edu; ashish747raj@gmail.com

1 Department of Surgery, Mayo Clinic, 200, $1^{\text {st }}$ St SW, Rochester, MN 55905, USA

2 Institute of Urology, Madras Medical College, 3, EVR Periyar Salai, Parktown, Chennai 600003, India

3 Department of Obstetrics and Gynecology, Singapore General Hospital, Singapore, Singapore
2. Hernia uteri inguinalis (as described by the authors) where one testes with its Mullerian attachment presents as an inguinal hernia

3. Transverse testicular ectopia with both testes herniating into a single processus vaginalis.

As the authors rightly pointed out, there is debate regarding optimal management of the primitive Mullerian structures [1,3]. While there seems to be scant evidence of malignancy and routine removal is not advocated, the removal of these structures when presenting as a hernia uteri inguinalis may be attempted while preserving the blood supply to the vas. While the authors' efforts to preserve the testis are commendable, it is imperative to counsel these patients and schedule routine ultrasound followup to evaluate for testicular malignancy. Additionally, patients should be encouraged to perform regular self-examination of the testis over their entire life span. We congratulate the authors in shedding light on this gray area and promoting discussion on this rare surgical condition.

\section{References}

1. Jha BK, Garg R, Soni RK, Singh K, Aneja H (2018) Hernia uteri inguinale: a travesty of nature. Indian J Surg 81(2):192-194. https:// doi.org/10.1007/s12262-018-1811-7

2. Jeong SC, Lee S, Ku JY, Lee SD (2014) Clinical characteristics and treatment of cryptorchidism in adults: a single center experience. World J Mens Health 32(2):110. https://doi.org/10.5534/wjmh. 2014.32.2.110

3. Rajesh A, Farooq M (2017) A rare case of male pseudohermaphroditism-persistent Mullerian duct syndrome with transverse testicular ectopia - case report and review of literature. Int J Surg Case Rep 37:72-75. https://doi.org/10.1016/j.ijscr.2017. 06.016

Publisher's Note Springer Nature remains neutral with regard to jurisdictional claims in published maps and institutional affiliations. 